this nomenclature by Dr. Kaiser, despite the fact that he and other German gas chromatographers had themselves independently drawn up a scheme (used in the German edition) which was in some points in conflict with the International Union of Pure and Applied Chemistry scheme (and arguably an improvement on it). However, a strong protest must be made against the use of "relative specific retention volume" (Volume 1, p. 26), which is an unnecessary development and a contradiction in terms. It is to be noted that Kaiser has used an alternative definition for 'resolution' and rightly takes the number of theoretical plates per metre as the measure of column performance. On another point in nomenclature, 'chromathermography' is used on p. 31 to mean the application of a temperature gradient to the column (as is most generally understood) whereas on p. 26 and elsewhere it appears to include the use of programmed temperature.

The translation is satisfactory, although here and there an odd expression occurs, which needs interpretation into more familiar gas chromatographic jargon, but seldom causes confusion. No attempt has been made to change the German background of the work, but this is no serious disadvantage and may be of interest to the reader in Great Britain. A few points for detailed criticism were noted: on p. 46 (Volume 1) we learn that "home-made support with optimum pores and minimum pressure drop is also very good", but we look in vain for instructions how to make it; on p. 137 a relay circuit includes no relay (circuits are all given in what is presumably the German convention and unfamiliar in Great Britain); on p. 15 a pressure correction factor is quoted to five figures (the more realistic three figures are given in the table in Volume 3); on p. 140 the thermal insulating properties of foam-glass are extolled, but it is my experience that glassfibre is to be preferred; in Fig. 22 (p. 77) the height for $\Delta p$ is shown incorroctly.

The format of the books is good, but gives rise to a reflexion on the economics of book production. The German edition was in paper-backs, at a total cost of about one of these volumes. At that price, every gas chromatographer, experienced or beginner, could be recommended to buy his own set, but at the present price it is obvious that he will not. However, the three-volume form does at least allow him to buy the part he is most interested in, and the library set can be split among several readers-of which there should be many.

D. Ambrose

\section{CONTRIBUTIONS TO AERODYNAMIC THEORY AND EXPERIMENT}

The Scientific Papers of Sir Geoffrey Ingram Taylor

Edited by G. K. Batchelor. Vol. 3 : Aerodynamics and the Mechanics of Projectiles and Explosions. Pp. xii +559. (Cambridge: At the University Press, 1963.) $100 s$.

VOT

OLUME 3 of the collected works of Sir Geoffrey Taylor contains fifty-eight papers published between 1910 and 1957, and concerned with various aspects of aerodynamies. The most notable papers deal with compressible-flow theory. Twenty-four of the papers appear here for the first time and are the fruits of Sir Geoffrey's war effort. Their addition to the scientific literature gives a continuity to Sir Geoffrey's work which has hitherto been lacking.

The uniform distribution of these papers in time shows the author's continued interest in this field throughout his very busy life. A continuity of a different kind is also apparent within this volume. These papers span the period of development of the theory of compressible flow as it is known to-day. The first paper, which is the now famous contribution to that most fundamental problem in compressible flow, the structure of a shock-wave, emphasizes this continuity with the roots of the subject in a reference to "A recent note" by Lord Rayleigh.

The papers are not now of equal interest, but the reader will find many interesting comments to entertain and stimulate him, and often in the most unlikely places. The Wilbur Wright Lecture for 1921 on "Scientific Methods in Aeronautics" is as relevant to-day as when it was delivered. Although the problems of scientific administration could not have been so pressing then as they are to-day, the solutions offered are now too familiar to need repeating. Whichever paper is considered, the reader will find a clarity of expression and a directness of approach to the problem in hand which make it worthy of examination. In war-time or peace-time, the practical nature of the problem to be solved is always emphasized. The powerful techniques of mathematical analysis which the author has at his disposal are never allowed to obscure the solution of the real problem. This ruthlessness of approach welds theory and experiment together in a unique way in these papers.

Probably the best-known papers in this volume are those on "The Air Pressure on a Cone moving at High Speeds, I and 2" (with J. W. Maccoll), "The Formation of a Blast Wave from a Very Intense Explosion, 1 and 2", "The Instability of Liquid Surfaces when Accelerated in a Direction Perpendicular to their Planes", and the paper already mentioned, "The Conditions Necessary for Discontinuous Motion of Gases". The first of these, now familiar as the "Taylor-Maccoll Solutions", contains the theoretical solution to the problem of supersonic flow past a cone. In the form of tables published by the Massachusetts Institute of Technology, these solutions have become a standard for the testing of both theoretical and experimental techniques in supersonic flows. The advent of the atomic bomb in the New Mexico explosion of 1945 vindicated the theoretical investigations set out in the second of these papers. Although the main features of the solution for a point explosion are here derived, the author had to resort to numerical methods to obtain the final result. The complete analytic solution to the problem has now been given by Latter and Sedov. The third paper deals with the generalization of the surface instability observable in an upturned tumbler of water. Such an instability occurs when an interface is accelerated in a direction from the lighter to the heavier fluid. It is now referred to as "Taylor Instability".

The papers contained in this volume, although collected from many sources, have been standardized and the figures redrawn in a uniform style. The whole is presented in the polished manner we have come to expect from the editor and publisher alike.

N. C. Freeman

\section{DELAYED IMPLANTATION}

Delayed Implantation

Edited by Allen C. Enders. Pp. $x+318$. (Published for William Marsh Rice University by the University of Chicago Press, Chicago, 1963.) 63s.

DELAYED IMPLANTATION comprises the proceedings of a symposium held at Rico University in 1963. The contributors were evidently selected with care and the papers have been arranged in logical sequence, avoiding the haphazard nature of many volumes of this genre. The book remains, however, a collection of specialist papers, and contains no general account of delayed implantation. The editor's prefaco is brief and formal, and the introductory address by C. G. Hartman and the summary of the conference by E. C. Amoroso are both modest in scope.

One may question the extent to which delayed implantation constitutes a study in itself. All mammalian embryos appear to pass through a quiescent phase after 